\title{
Results from bone mineral density scans in twenty-two complete lesion paraplegics
}

\author{
DE Wood*,1, AL Dunkerley $^{1}$ and AM Tromans ${ }^{2}$ \\ ${ }^{1}$ Department of Medical Physics and Biomedical Engineering, Salisbury District Hospital, Salisbury, Wiltshire, UK; \\ ${ }^{2}$ Duke of Cornwall Spinal Treatment Centre, Salisbury District Hospital, Salisbury, Wiltshire, UK
}

\begin{abstract}
Study design: The bone mineral density (BMD) in 22 male subjects with complete lesion paraplegia sustained 1.8 to 27 years previously was measured. The measurements were used in screening each subject for a research programme investigating the restoration of standing using functional electrical stimulation (FES).
\end{abstract}

Objectives: To assess the extent of bone loss in this group of subjects and correlation to age, time post-injury and level of lesion.

Setting: District General Hospital in the UK.

Methods: BMD was measured by dual energy X-ray absorptiometry (DEXA) in the lumbar spine and femoral neck and expressed as an indirect index to an age matched 'normal' population. Fracture risk was described from this score using published data indicating that the risk increased with each standard deviation difference from the 'normal' mean.

Results: The bone density in the lumbar spine was better preserved than in the femoral neck. BMD in the lumbar spine was found to be greater than the mean from the age matched population in $57.1 \%$ of subjects. Bone loss at the femoral neck suggested that $81.8 \%$ of the subjects were at increased risk of fracture, but only $22.7 \%$ were at a high risk. No correlation was found between BMD at the lumbar spine or the femoral neck and age, lesion level or time post-injury.

Conclusion: The study indicates that further investigation into baseline BMD values for the SCI population is required to improve information provided to patients and assessment of fracture risk on an individual basis.

Sponsorship: U.K. Medical Research Council and the Wellcome Trust.

Spinal Cord (2001) 39, $145-148$

Keywords: bone mineral density; paraplegia; spinal cord injury; fracture

\section{Introduction}

It is well known that immobilisation leads to a reduction in bone mineral density (BMD), that can lead to an increased risk of fracture. ${ }^{1}$ Spinal cord injury (SCI) is no exception. In the first months following SCI, there is a rapd loss of BMD in sublesion bones. ${ }^{2,3}$ Though BMD reaches a new steadystate level at approximately 2 years, these levels are below normal values; pelvis $55 \%-60 \%,{ }^{3}$ femoral neck $60 \%-70 \%$ and proximal tibia $40 \%-50 \%{ }^{4}$ of normal. It is recognised that collecting normal values for BMD in SCI subjects would be helpful to estimate the individual fracture risk and to decide on the necessity to treat any bone loss. ${ }^{5}$

*Correspondence: DE Wood, Department of Medical Physics and Biomedical Engineering, Salisbury District Hospital, Salisbury, Wiltshire SP2 8BJ, UK

\section{Study design}

The BMD in 22 male Caucasian subjects who had suffered a traumatic complete SCI was measured. Measurement of BMD was used in screening subjects for a research programme investigating the restoration of standing using functional electrical stimulation (FES). ${ }^{6}$ Subjects were aged between 21 to 54 years (mean 36.4, standard deviation 10.0), with thoracic lesion levels between $\mathrm{T} 3$ to $\mathrm{T} 12$. In all subjects it was postulated that the BMD was in equilibrium, with times post-injury being from 1.8 to 27 years (mean 7.6, standard deviation 6.8). No subjects demonstrated excessive spasticity and all had good range of movement around the hips. None showed any clinical signs of heterotropic ossification or were known to be suffering from any metabolic disease that may have influenced calcium metabolism. 
BMD of the left femoral neck and the lumbar spine (L1-L4) was measured using dual energy X-ray absorptiometry (DEXA) at two centres. Both centres used a Hologic (Vertec Scientific Ltd., 5 Comet House, Calleva Park, Aldermaston, Reading RG7 8JA, UK) scanner - one using the QDR-1000 on nine of the 22 subjects, and the other using the QDR-2000 on the remaining 13 subjects. Each scanner is serviced every 6 months and daily quality checks are conducted to ensure that errors in the measurement of bone mineral content and geometry are less than $1 \%$. Precision values for both QDR systems are $1.2 \%$ for the spine and $1.5 \%$ for the femoral neck.

Bone integrity was assessed using an indirect index, the Z-score, calculated from the absolute BMD. This index expresses the deviation of the BMD from the mean of an age matched 'normal' population in terms of standard deviations. Large negative scores are indicative of a low BMD, and this has been shown to be associated with an increased risk of fracture. Results from studies suggest that for a 2 standard deviation difference in bone mass there is a $2-4$-fold increase in fracture risk at non-spine sites ${ }^{7}$ and a 4-6fold increase at vertebral sites. ${ }^{8}$ Though it is recognised that these are predominantly from postmenopausal women, we have used the above to describe benchmarks of fracture risk since very few studies have been conducted either on men or on paraplegics. In discussing our results, we use $\mathrm{Z}<-1$ to indicate bone that may be at an increased risk of fracture and $\mathrm{Z}<-2$ that at a high risk of fracture.

\section{Results}

Of the 22 subjects, the BMD at the femoral necks in 21 $(95.5 \%)$ subjects were below the normal mean $(Z<0)$. The Z-score suggested that eighteen $(81.8 \%)$ of the subjects were at increased risk of fracture $(Z<-1)$, but only five $(22.7 \%)$ were at a high risk $(Z<-2)$ (Figure $1)$. The DEXA images did not show any evidence of heterotropic ossification at the hip. There was found to be no strong correlation between the Z-score and age (correlation coefficient, $r=-0.01$ ), lesion level $(r=+0.59)$ and time post-injury $(r=-0.14)$.

For the lumbar spine, the BMD was measured in 21 subjects. However, because of internal fixation in five subjects, the data for L1-L4 is only shown in sixteen subjects (Figure 2). Only one $(4.8 \%)$ subject was shown to be at risk of fracture $(\mathrm{Z}<-1)$, and twelve $(57.1 \%)$ had BMD above the normal mean $(Z>0)$. Again there was found to be no strong correlation between the Z-score and age $(r=+0.12)$, lesion level $(r=+0.29)$ and time post-injury $(r=+0.45)$.

\section{Discussion}

The BMD in the lumbar spine was better preserved than in the femoral neck, in agreement with other studies. ${ }^{4,9-11}$ This is not surprising, considering the weight bearing role and impact through the lumbar spine during wheelchair transfers in this group of subjects. The measurements from the femoral neck also supported the results from other studies, that the BMD following SCI is less than normal values. ${ }^{4,10-12}$ However, our results suggested that bone loss is less than what is generally perceived by therapists and the SCI population and raises some issues. The finding that BMD loss is not correlated with time post-injury is not in agreement with another study with paraplegics. ${ }^{13}$ This may be explained that their patients were at the more acute stage (mean 9.5 months, range 2-30 months) perhaps before bone loss had reached a steady state. Lack of correlation to age and level of injury was in agreement between both studies.

The authors recognise that DEXA images cannot be used in diagnosis, but we believe that heterotropic ossification has been ruled out from examination of these images and from clinical assessment. It may be argued that this group of subjects is not representative of the general SCI population, because each one had volunteered to participate on a research programme to investigate standing by electrical stimulation. It is true to say that all had completed a rehabilitation
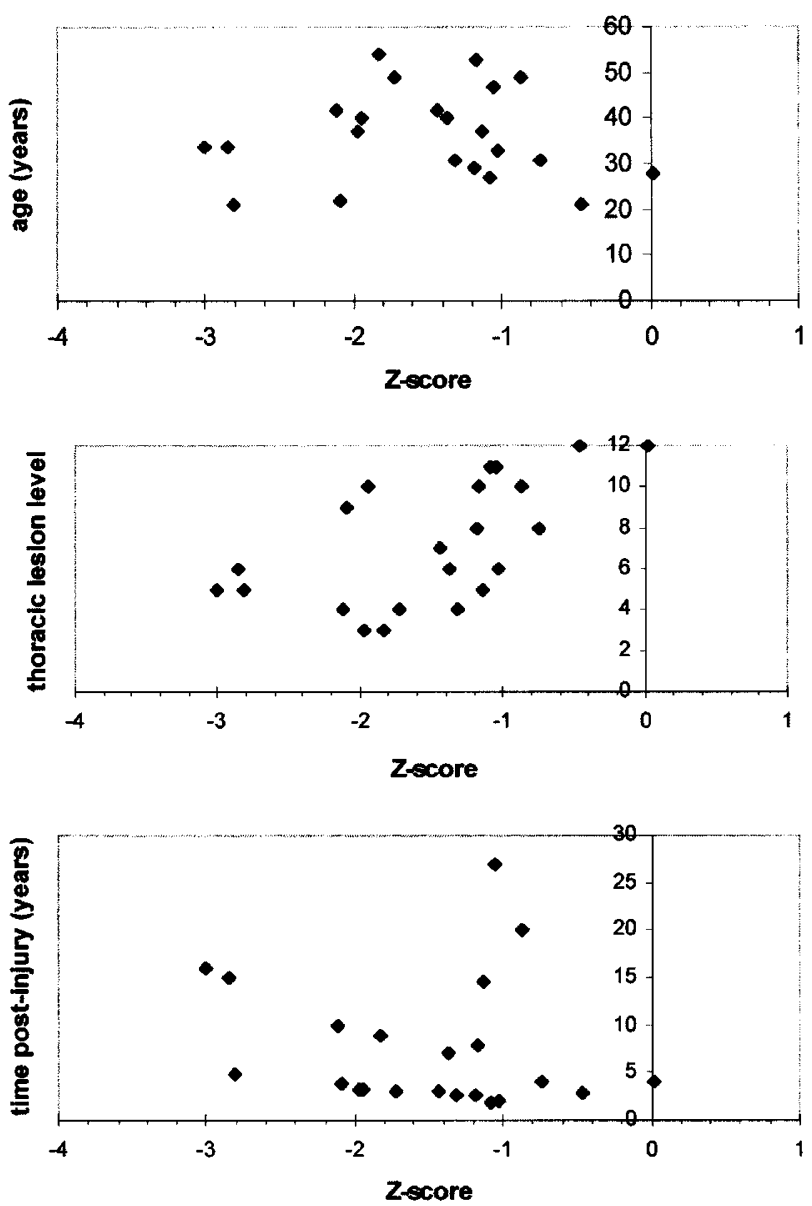

Figure 1 Z-scores for the femoral neck 
programme following their injury and, during their rehabilitation, all had tried weight-bearing exercises, by standing passively and/or using calipers. However, there was no evidence to suggest that their use of these exercises either during rehabilitation or post-discharge was different to the general SCI population. Another possible factor may have been the time post-injury, taking into account the changes in rehabilitation management over the last 10 or so years, encouraging early mobilisation of SCI persons. Our result examining the correlation between the time postinjury and the BMD suggests that this is not the case.

It is known that fractures of the lower extremities from minor trauma are more prevalent in the SCI than in the non-SCI population, $2 \%$ compared to $1 \%$ per year. ${ }^{14}$ However, these fractures may be as related to activities of the patients, as much as to the quality of the bone. This is borne out in clinical practice at Salisbury District Hospital, where the fractures seen tend to be from patients with X-rays that suggest the presence of good quality bone, but they lead a more aggressive lifestyle and thus sustain the injury. Despite this, SCI patients are still encouraged by therapists to stand passively to minimise the development of
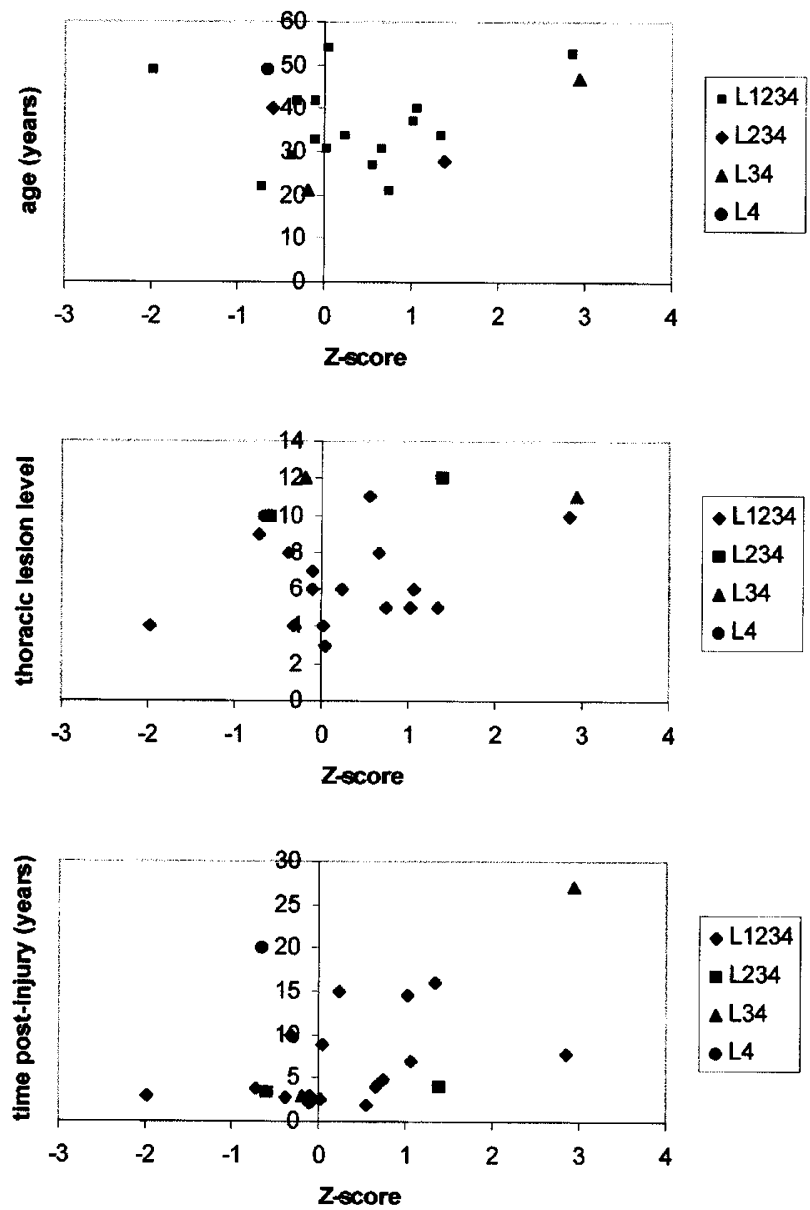

Figure 2 Z-scores for the lumbar spine osteoporosis of the long bones and reduce the risk of fracture. ${ }^{15}$ Though standing is recognised to be important for other reasons, the evidence to support the above claim is inconclusive. ${ }^{11,16}$

It is recognised that this study is based on a small number of subjects, but we believe the results indicate that a systematic approach is required to address these issues, though we acknowledge that assessing fracture risk solely from BMD measurements is only part of this. First, routine measurements of BMD, something as far as we are aware that is not done in spinal units, are required to establish a new baseline for this group of subjects. Secondly, an audit of fracture incidences in the SCI population and identifying specific traumas, such as falls from chairs. Thirdly, to conduct serial measurements from paraplegics, following them over many years. This can then be used to improve both the information provided to patients and assess the fracture risk for each subject, based on data applicable to this population, taking into account individual lifestyle and activities.

\section{Conclusion}

Further investigation into baseline BMD values for the SCI population is required to improve information provided to patients on rehabilitation modalities.

\section{Acknowledgements}

The authors would like to thank the U.K. Medical Research Council and the Wellcome Trust for funding these scans, the radiographers at the Royal National Orthopaedic Hospital and Southampton General Hospital, and Vishna Shah (Director, Metabolic Unit, Royal National Orthopaedic Hospital Trust) for her helpful suggestions during the study.

\section{References}

1 Uebelhart D, Demiaux-Domenech B, Roth M, Chantraine A Bone metabolism in spinal cord injured individuals and in others who have prolonged immobilisation - a review. Paraplegia 1995; 33: $669-673$.

2 Garland D et al. Osteoporosis after spinal cord injury. $J$ Orthopaed Res 1992; 10: $371-378$.

3 Wilmet $\mathrm{E}$ et al. Longitudinal study of the bone mineral content and of soft tissue composition after spinal cord section. Paraplegia 1995; 33: 674-677.

4 Biering-Sorensen F, Bohr H, Schaadt O. Longitudinal study of bone mineral content in the lumbar spine, the forearm and the lower extremities after spinal cord injury. Eur J Clin Invest 1990; 20: $330-335$

5 Reiter A et al. Osteoporosis in male patients with spinal cord injury: correlation with clinical factors. In: Proceedings of the 37th Annual Scientific Meeting of IMSOP 1998. Iguassu, Brazil.

6 Rushton D et al. Selecting candidates for a lower limb stimulator implant programme - a patient centred method. Spinal Cord 1998; 36: $303-309$.

7 Ross P, Davis J, Vogel J, Wasnich R. A critical review of bone mass and the risk of fractures in osteoporosis. Calc Tiss Int 1990; 46: $149-161$ 
8 Ross P, Davis J, Epstein R, Wasnich R. Pre-existing fractures and bone mass predict vertebral fracture incidence in women. Ann Int Med 1991; 114: 919-923.

9 Pacy $\mathrm{P}$ et al. Muscle and bone in paraplegic patients and the effect of functional electrical stimulation. Clin Sci 1988; 75: $481-487$.

10 Szollar S et al. Demineralization in tetraplegic and paraplegic man over time. Spinal Cord 1997; 35: $223-228$.

11 Goemaere S, van Laere M, de Neve P, Kaufman J. Bone mineral status in paraplegic patients who do or do not perform standing. Osteopor Int 1994; 4: $138-143$.

12 Chow Y et al. Ultrasound bone densitometry and dual energy Xray absorptiometry in patients with spinal cord injury - a cross sectional study. Spinal Cord 1996; 34: 736-741.
13 Demirel G, Yilmaz H, Paker N, Onel S. Osteoporosis after spinal cord injury. Spinal Cord 1998; 36: $822-825$.

14 Vestergaard P, Krogh K, Rejnmark L, Mosekilde L. Fracture rates and risk factors for fractures in patients with spinal cord injury. Spinal Cord 1998; 36: 790-796.

15 Bromley I. Tetraplegia and Paraplegia - A guide for physiotherapists. 5th edn (ISBN 0-443-05872-5). 1998 Edinburgh: Churchill Livingstone.

16 Biering-Sorensen F, Bohr H, Schaadt O. Bone mineral content of the lumbar spine and lower extremities years after spinal cord lesion. Paraplegia 1988; 26: $293-301$. 